\section{Evidence that hand osteoarthritis is linked with comorbidities}

Osteoarthritis $(\mathrm{OA})$, in addition to being one of the most frequent morbidities in the elderly, has been associated with a high rate of comorbidities. Kalichman and colleagues have investigated the extent to which chronic morbidity is associated with hand OA in an unusual population-the occupants of small villages in the Republic of Chuvashia, Russian Federation.

All 819 participants were interviewed about their health status, and their medical records were assessed. The presence and extent of OA was assessed via radiographs of both hands. Of the 14 categories of chronic morbidity investigated, only two were found to have a statistically significant association with age-adjusted hand OA score: ischemic heart disease and gastrointestinal diseases $(P=0.022$ and $P=0.043$, respectively). Participants with ischemic heart disease were more likely to have hand OA than were participants without heart disease, and participants with a gastrointestinal disease were less likely to have hand OA than were participants without such a disorder.

The latter finding was unexpected; previous studies have found gastrointestinal disease to be more, rather than less, common in OA sufferers who were taking NSAIDs. The participants of Kalichman et al.'s study, however, were largely untreated. The authors suggest that the study participants with gastrointestinal diseases might follow a diet that discourages joint degeneration. Further study is recommended, so that strategies to diagnose and prevent OA-associated morbidity can be developed.

Original article Kalichman L et al. (2006) The association between morbidity and radiographic hand osteoarthritis: a population-based study. Joint Bone Spine 73: 406-410

\section{Ethnic variation in MTHFR polymorphisms in patients with RA}

Methylenetetrahydrofolate reductase (MTHFR) has an important role in folate metabolism, and could affect a patient's response to the antifolate drug methotrexate. Previous studies of the MTHFR gene have indicated ethnic differences in allele frequency for certain MTHFR single-nucleotide polymorphisms (SNPs). A new study by Hughes and colleagues has now confirmed that MTHFR allele frequencies differ between white and African American patients with rheumatoid arthritis (RA) and that MTHFR genotype can affect response to methotrexate treatment.

The study included 393 white and 138 African American patients with RA, and 50 white and 53 African American healthy controls. Hughes and colleagues assessed five MTHFR SNPs: the allele frequencies of the rs $4846051 \mathrm{C}$, $677 \mathrm{~T}$, and $1298 \mathrm{C}$ alleles were $0.08,0.30$, and 0.34 , respectively, among white RA patients, compared with $0.33,0.11$, and 0.13 , respectively, among African American patients. There were no differences in the frequencies of the other two alleles assessed. There were also no differences in allele frequency between patient and control groups, regardless of ethnicity. Although the efficacy of methotrexate treatment was not affected by allele frequency, toxicity of the drug was: the 1298A allele was associated with an increased likelihood of adverse events in white patients, and the rs4846051C was associated with increased toxicity in African American patients.

The authors conclude that ethnic differences in allele frequency do exist, and call for larger trials, as their analysis might have been limited by the relatively small number of African Americans in their study.

Original article Hughes LB et al. (2006) Racial or ethnic differences in allele frequencies of single-nucleotide polymorphisms in the methylenetetrahydrofolate reductase gene and their influence on response to methotrexate in rheumatoid arthritis. Ann Rheum Dis 65: 1213-1218

\section{Genetic versus socioeconomic causes of ESRF in black patients with lupus nephritis}

A UK study has concluded that the higher rate of end-stage renal failure (ESRF) observed in black patients compared with white patients with lupus nephritis is probably caused by genetic factors rather than by socioeconomic status. Previous studies in the US also found an elevated rate of ESRF in black patients, but could not distinguish between the relative contributions of ethnicity and socioeconomic status.

This study identified 401 patients with systemic lupus erythematosus from the University College London Hospitals cohort. Between 